TITLE:

\title{
Dry galloping characteristics and its mechanism of inclined/yawed cables
}

\section{$\operatorname{AUTHOR}(S)$ :}

Matsumoto, Masaru; Yagi, Tomomi; Hatsuda, Hideaki; Shima, Takanori; Tanaka, Masanobu; Naito, Hiroko

\section{CITATION:}

Matsumoto, Masaru ... [et al]. Dry galloping characteristics and its mechanism of inclined/yawed cables. Journal of Wind Engineering and Industrial Aerodynamics 2010, 98(6-7): 317-327

ISSUE DATE:

2010-06

URL:

http://hdl.handle.net/2433/123452

\section{RIGHT:}

(C) 2010 Elsevier B.V.; This is not the published version. Please cite only the published version.; この論文は出版社版でありません。引用の際に は出版社版をご確認ご利用ください。 


\section{Title:}

Dry galloping characteristics and its mechanism of inclined/yawed cables

\section{Authors:}

Masaru Matsumoto 1)

Tomomi Yagi 1)

Hideaki Hatsuda 2)

Takanori Shima 3)

Masanobu Tanaka 4)

Hiroko Naito 1)

\section{Affiliations:}

1) Department of Civil and Earth Resources Engineering, Kyoto University, Kyotodaigaku-katsura, Nishikyo-ku, Kyoto 615-8540, Japan

2) Tokuyama Corporation, 1-1, Mikage-cho, Shunan city, Yamaguchi 745-8648 Japan

3) IHI Corporation, 1-1, Toyosu 3-chome, Koto-ku, Tokyo 135-8710, Japan

4) Penta Ocean Construction Co., Ltd., 2-8 Koraku 2-chome, Bunkyo-ku, Tokyo 112-8576, Japan

\section{Corresponding Author:}

Masaru Matsumoto

Department of Civil and Earth Resources Engineering, Kyoto University,

Kyotodaigaku-katsura, Nishikyo-ku, Kyoto 615-8540, Japan

TEL: $\quad+81753833165$

FAX: $\quad+81753833168$

E-mail: matsu@brdgeng.gee.kyoto-u.ac.jp 


\begin{abstract}
Mechanism of dry galloping of inclined cable of cable-stayed bridges is described in relation to Karman vortex mitigation. Furthermore, the role of Scruton number Sc on reduced critical velocity Vrcr of the dry galloping is investigated for practical use basing on wind tunnel tests and field observations of dry galloping or pseudo-galloping, which is classified as cable vibration with rain-state but response amplitude is abnormally large. It is verified that as far as the divergent-type of dry galloping, the design criterion subject to Sc-Vrcr proposed by FHWA (Federal Highway Administration of U.S.) seems to be reasonable for practical use, on the other hand, for the unsteady dry galloping, the Saito criterion for Sc-Vrcr diagram seems to be reasonable.
\end{abstract}

\title{
Keywords:
}

inclined cable aerodynamics, dry-galloping, role of Karman vortex, quasi-steady galloping, unsteady galloping 


\section{Introduction}

The complicated inclined cable aerodynamics has been clarified through a lot of wind tunnel tests and researches. As a state of art of inclined cable aerodynamics, it can be understood that rain vibration is caused by formation of upper water rivulet at particular position on cable-surface and axial flow in near wake of cable (Matsumoto, 1998; Matsumoto et al., 1992, 2005). On the other hand, as far as dry galloping, the axial flow and critical Reynolds number Recr play definitely important role for the excitation (Cheng et al., 2003; Larose et al., 2003). Taking into account the common characteristics of mitigation of Karman vortex shedding by the upper water rivulet at the particular position, the existence of intensive axial flow in near wake and the critical Reynolds number, it is indicated that aerodynamic response of inclined cable, including galloping, might be highly related with mitigation of Karman vortex. Galloping generation mechanism has been explained as the appearance of inner circulatory flow on side surface by aerodynamic interference between separated flow from leading edge and sharp trailing edge by Bearman et al. (1972), and appearance of reattachment-type pressure on side surface by interruption of two shear layers by Nakamura et al. (1994). Furthermore, it is known that if the time-averaged flow would reattach on side surface, galloping never appears. Therefore galloping of rectangular cylinder disappears at the particular side ratio of 2.8 that is upper limit. On the other hand, the lower limit of side-ratio is 0.8 . At the range of side-ratio of less than 0.8 , galloping does not occur. This is caused by intensive Karman vortex shedding at less than this particular side-ratio of 0.8. From these stability conditions against galloping, its instability can be also related to the intensity of Karman vortex. Thus, the role of Karman vortex on galloping for the bluff bodies with non-reattachment of separated flow from leading edge is 
clearly implied. However, at present, the detail and general effects of Karman vortex on galloping instability has not been clarified.

Sometimes, significantly sever cable vibrations have been observed in the field for proto-type cable-stayed bridges. How to stabilize these cable vibrations is one of the most important safe design factors for bridges. The damping devises to increase structural damping (Matsumoto, 2000; Matsumoto et al., 1994) or modification of cable geometrical shape have been so far used to control the cable vibration (Saito et al., 1994; Matsumoto et al., 2007). Recently in U.S. to achieve this matter, three different countermeasures are simultaneously used (FHWA/HNTB, 2005). Those are cables lapped by polyethylene with helical fin on cable surface, cross ties between stay-cables and damping devices. In particular, how to determine the damping capacity to sufficiently suppress the dry galloping is the most concerned. Then, FHWA in U.S. (FHWA/HNTB, 2005) proposed the original diagram of reduced critical velocity Vrcr Scruton number Sc, so called as FHWA criterion as shown in Fig.1 to determine the required damping capacity for practical use. However, Saito et al. (1994) formerly reported different wind tunnel test results associated to Sc-Vrcr diagram (see Fig.1), so called Saito criterion. As shown in Fig.1, there is a great difference between two criteria, in another expression, basing on Saito criterion, it is really difficult to suppress the dry galloping by increasing structural damping. The clarification of definite discrepancy between two criteria is one of the most important issues in latest bridge aerodynamics. If using recent finding by one of authors on galloping instability of bluff body aerodynamics in relation with role of Karman vortex, the key to resolve this problem is found. Furthermore, the galloping would be classified into two different types, those are 
the divergent type quasi-steady galloping and the unsteady galloping, depending on the controlled Karman vortex characteristics of bluff bodies (Matsumoto et al., 2007).

On the other hand, the mechanism of galloping of bluff bodies has been studied by Nakamura et al. (1994), and he concluded that the interruption of "communication" between two separated shear layers in near wake can produce the reattachment-type of pressure distribution and it can excite galloping instability.

In this paper, the dry galloping of inclined cables being targeted, how to control the dry galloping and consideration on its mechanisms are studied basing on a series of wind tunnel tests.

\section{Field observation of dry galloping for proto-type cables}

The wind-induced vibration of inclined cables of cable stayed-bridges have been mostly observed under the state of precipitation, that is well known as the rain-wind induced vibration. However, the wind tunnel tests have reported the galloping instability of inclined/yawed cable at the dry state, it means without rain.

Recently, in Japan, an inclined polyethylene-lapped cable with $187 \mathrm{~m}$ length of certain cable-stayed bridge showed a violent vibration, during passing of typhoon, whose amplitude was estimated more than $1.5 \mathrm{~m}$, and this vibration severely damaged not only a part of edge faring installed at bridge girder edges but also cable surface, as shown in Fig.2 (Matsumoto et al., 2005). The estimated wind velocity was approximately $18 \mathrm{~m} / \mathrm{s}$ and wind blew with some yawing angle to bridge axis, which were not precisely measured on the bridge site but based upon the data measured at a meteorological observatory located at approximately $1 \mathrm{~km}$ upstream side from bridge. 
Also, some eyewitness reported that rain had already stopped when the violent cable vibration started. The viscous damper had been installed at the cable-end on the ground, because of curved bridge-girder, but it was completely destroyed by the violent cable vibration. However, it was not cleared whether this damper was damaged before or after the violent cable vibration. Therefore, this vibration might be a dry-state galloping, even though less detail data at the bridge site on that day.

\section{Axial flow effects on inclined cable aerodynamics}

One of authors has pointed out the important role of axial flow in a wake of inclined/yawed cable without rivulet, in another expression at the dry-state, for galloping instability (Matsumoto, Yokoyama et al., 1989). Axial flow velocity in near wake has been measured by use of hot-wire anemometer for yawed rigid cable model in the wind tunnel and it became clear that the axial flow velocity increased with yawing angle $\beta$ to approaching wind. Furthermore, this velocity distributes non-uniformly along cable axis from the upstream cable end to the downstream one. Three kinds of cable-end conditions were tested, such as (1) free cable ends and without tunnel-walls, (2) with end-plates and without tunnel-walls and (3) with tunnel-walls installed suitable holes, so called "window", at the cable ends. In order to survey the effect of cable end condition on the axial flow velocity, its velocity was measured in the wake for three different cable end cases. The intensity of those particular flow velocities is not so different in the three end conditions, except at near upstream cable end, as shown in Fig.3 ( $V=8 \mathrm{~m} / \mathrm{s}$, $\beta=45^{\circ}$, in smooth flow). At near the upstream cable end, the axial flow velocity is almost $80 \%$ for the end condition case (3), $70 \%$ for the case (1) and $50 \%$ for the case (2) 
of approaching wind velocity, respectively. However, from the quarter point of cable length to the downstream side, its intensity varies $60 \%$ to $40 \%$ with the trend of gradually decreasing to the downstream end for all three end conditions. On the other hand, the axial flow was visualized in the field by use of light strings for the proto-type inclined cable with vertical angle $\alpha$ of $30^{\circ}$, which corresponds to equivalent yawing angle (Matsumoto et al., 1990), $\beta^{*}$ of $40^{\circ} \sim 50^{\circ}$, from the point of relative cable attitude to wind, as shown in Fig.4. Its velocity was measured by a soap-bubble movement in the wake taken by video camera. The measured axial flow velocity distributed between $40 \%$ and $80 \%$ to approaching wind velocity, as shown in Fig.5, which corresponds well to wind tunnel test results explained before.

The artificial axial flow, generated by compressor and electrical cleaner as shown in Fig.6, can reproduce galloping instability for non-yawed $\left(\beta=0^{\circ}\right)$ cable model significantly, which onsets at the particular reduced velocity of $V r=V / f D=40$ similarly with one of yawed cable model with $\beta=45^{\circ}$, see Fig.7 (Matsumoto, Knisely et al, 1989). This test result indicates clearly that the axial flow in near wake can excite galloping. Taking account the similar role of axial flow with a splitter plate installed in near wake, the axial flow must interrupt Karman vortex shedding in near wake. However, V-A (velocity-amplitude) diagram of cross-flow response indicated different characteristics as shown in Fig.8, because the intensity of axial flow changes along yawed-cable $\left(\beta=45^{\circ}\right)$ axis, depending on the cable end-conditions, including with free end, with end-plates and with suitable-size window on wind-tunnel wall.

\section{Critical Reynolds number effects on inclined cable aerodynamics}


Schewe (1983) pointed out that drag force is remarkably reduced, stationary lift force can be generated and Karman vortex shedding is significantly suppressed for a circular cylinder at critical Reynolds number. Furthermore, Cheng et al. (2003) reported that inclined cable model $\left(\beta=0^{\circ}\right.$ and $\left.\alpha=45^{\circ}\right)$ also produces stationary lift force with significant drag reduction at critical Reynolds number. Macdonald (2005) successfully realized the galloping instability by use of quasi-steady analysis basing on test result obtained by Cheng. On the other hand, the authors verified the critical Reynolds number effect by use of non-yawed cable model with artificial roughness on galloping instability. Also, in this case, the stationary lift force appearance, significant drag reduction and Karman vortex suppression at critical Reynolds number can be seen in Fig.9. The cross flow response is not divergent type galloping but unsteady response can be observed at critical Reynolds number as shown in Fig.10. Furthermore, basing on Scanlan's derivative $H_{1}{ }^{*}$ in terms of heaving velocity, it shows positive value, which means unstable state for galloping, at critical Reynolds number, as shown in Fig.11. These values can be defined by particular combination of wind velocity and forced vibration frequency.

\section{FHWA Criterion and Saito Criterion associated with Scruton number and} galloping onset reduced velocity characteristics

As described above, recently FHWA proposed a new criterion on Scruton number and galloping onset reduced velocity characteristics for the dry galloping (FHWA/HNTB, 2005) for practical use for bridge designers. However, there are significant difference at large Scruton number between FHWA Criterion and the 
characteristics formerly reported by Saito as shown in Fig.1. As mentioned above, basing on Saito criterion, it is really difficult to suppress dry galloping by increasing structural damping. The amplitude of galloping fitted by Saito's criterion at large Scruton number is unsteady and not so large. Therefore, from the practical point of view, this unsteady galloping might not interfere directly with the safety of inclined cables. However, the clarification of definite discrepancy between two criteria must be one of the important issues in bluff body aerodynamics. The detail mechanisms of the unsteady galloping will be discussed later.

\section{Similarity between rain vibration and dry galloping}

For the rain vibration, extremely important role of formation of upper water rivulet at particular position on yawed/inclined cable surface for aerodynamic excitation has been known. As shown in Fig.12, galloping appears if Karman vortex is significantly suppressed by water rivulet formation at particular position for both of non-yawed cable $\left(\beta=0^{\circ}\right)$ and yawed cable $\left(\beta=45^{\circ}\right)$. In consequence, there is significant similarity between rain vibration and dry galloping from points of galloping appearance in relation with suppression of Karman vortex. However, taking into account of movement and non-uniform-distribution of upper water rivulet during cable vibration as shown in Fig.13, the galloping with large amplitude can be hardly excited due to formation of upper water rivulet, as alternatively beat vibrations like snake dance have been mostly observed in the field. As additional similarity between rain vibration and dry galloping, both phenomena for yawed/inclined cable can be excited by axial flow. Considering the extremely violent vibration of proto-type inclined cable observed in the field, it might 
be rather difficult to distinguish rain vibration and dry galloping, aerodynamically. Basing on the generation mechanism of galloping excited in relation with Karman vortex mitigation points of views, the differences of both vibrations are in the climate-conditions, which are with rain or without rain. In conclusion, there might be two different phenomena in cable aerodynamic vibrations observed in rainy and windy day. One is a typical rain vibration whose vibration mode is beat phenomenon like snake-motion, and it is a velocity and amplitude-restricted vibration, mostly observed in the fields. The other one is a significantly violent vibration like divergent-type galloping, which peak-to-peak amplitude must be up to more than $2.0 \mathrm{~m}$. The former one is definitely affected by formation of upper water rivulet and the later one might be an identical phenomenon of divergent dry galloping from the point of axial flow.

\section{Galloping generation mechanism}

Nakamura et al. (1994) pointed out that the generation mechanism of galloping is interruption of communication between upper and lower separated flows. Because the communication of two separated flows can tend to cancel pressure difference on upper and lower surfaces of cylinder. This interrupting communication between two separated flows can be accomplished by following three cases; (1) a long downstream splitter plate; (2) vanishing effect of wake undulation at low wind velocity related with low speed galloping; (3) critical geometry at high wind velocity which can produce a reattachment-type pressure distribution caused by separated-flow/edge interaction related with high speed galloping. Taking into account that the Karman vortex would be produced by communication of upper and lower separated flows, in another expression, 
the Karman vortex shedding should promote the communication between two separated flows, the interruption of this communication between two separated flows should be identical to the interruption of Karman vortex shedding. Therefore, it can be explained that mitigation or suppression of Karman vortex can excite galloping instability.

\section{Divergent-type galloping and unsteady galloping}

Galloping can be classified into two different types; those are divergent-type galloping and unsteady galloping. The former one corresponds to well known conventional galloping, and its response characteristics can be explained by quasi-steady theory. If Karman vortex is sufficiently suppressed in stationary state, the separated flow is released from the control of Karman vortex, then separated flow is so sensitive against external disturbance or stimulation, such as body motion, fluctuating coming-flow, applied sound and so on. Therefore, the mechanism of divergent-type galloping is appearance of motion-induced flow field, which is released from Karman vortex influence. During downward motion of cable, the lower-side separated flow approaches to cable surface, on the contrary, the upper separated flow leaves from cable surface. Then, down lift can be generated and self-excited vibration appears. From the point of flow field, this galloping mechanism is substantially identical with the one of low speed galloping of bluff body with splitter plate studied by Nakamura et al. (1991).

On the other hand, the unsteady galloping can be explained as follows: if Karman vortex mitigation is not sufficient in stationary state, cross flow response shows unsteady response with non-stationary amplitude. When Karman vortex is mitigated, response amplitude becomes large, on the contrary when Karman vortex sheds, 
amplitude becomes small. To confirm these characteristics a perforated splitter plate (see Fig.14) is installed in wake center of non-yawed $\left(\beta=0^{\circ}\right)$ circular cylinder, cross flow response varies with change of perforation ratio of splitter plate as shown in Fig.15. The perforation ratio of splitter plate can control the intensity of Karman vortex shedding from the body in stationary state. As decreasing perforation ratio, Karman vortex becomes weaker and galloping instability becomes more unstable, and then finally divergent-type galloping appears. As show in Fig.15, it should be noted that the maximum amplitude of vortex-induced vibration near resonant reduced velocity, $V r=1 / S t$, becomes larger as decrease of perforation ratio because of more mitigation of Karman vortex shedding. Also for yawed cable with smooth surface, the unsteady galloping can be observed by wind tunnel tests as shown in Fig.16 (described below in detail). In this case, Karman vortex shedding is unsteadily mitigated by axial flow and response amplitude varies according to the intensity of Karman vortex shedding.

As mentioned above, Karman vortex shedding around inclined/yawed cables can be mitigated by the axial flow, the critical Reynolds number effects and even its body motion. Then, the divergent-type galloping appears, if Karman vortex is sufficiently suppressed in stationary state, and this phenomenon can be described by quasi-steady theory. However, if Karman vortex is unsteadily mitigated by e.g. the axial flow with unsteady properties, then the unsteady galloping appears which response amplitude varies due to the fluctuations of Karman vortex intensity.

\section{Wind tunnel tests of galloping characteristics of yawed cable with $\beta=45^{\circ}$ in terms} of Scruton number $S c$ vs. reduced critical velocity $\operatorname{Vrcr}$ 
Under four different cable-surface conditions, those are smooth surface, with axial protuberances, helical fins and with rings, free vibration tests were carried out in smooth flow. The diameters of cable models are $50 \mathrm{~mm}$, and suitable size windows with $100 \mathrm{~mm}$ diameter holes on the both sides of wind tunnel walls were installed to promote axial flow in near wake. The measured cross-flow responses show unsteady galloping with unsteady amplitude or divergent-type galloping depending on Scruton number $S c$, as shown in Fig.17 for smooth surface cable. The other surface cases were fundamentally identical to ones of smooth surface case. As shown in Fig.16, the unsteady amplitude of cross flow response at velocity of $4 \mathrm{~m} / \mathrm{s}$ shows fairly good correlation with Karman vortex mitigation. If typical divergent-type galloping is not observed but the unsteady galloping appears, the reduced critical velocity is determined as the lowest reduced velocity where the double amplitude exceeds $40 \%$ of diameter $D$, which means $0.4 D$. If Scruton number $S c$ is enough small such as 1.22, divergent-type galloping was observed, which is caused by complete suppression of Karman vortex due to large vibration amplitude of itself. However, the majority of results indicate the unsteady galloping occurs under Sc larger than about 20. Fig.18 shows the tendency of Karman vortex mitigation due to the vibration amplitudes by measuring the unsteady lift forces during forced vibrations. It is clear that the Karman vortex component becomes weaker when the vibration amplitude becomes larger. These free vibration test results are plotted on the diagram of $\mathrm{Sc}$-Vrcr as shown in Fig.19. Furthermore, results of Scanlan derivative $H_{1}$ * obtained by forced vibration tests are also plotter on Fig.19. Scruton number $S c$ for onset wind velocity can be determined by $H_{1} *$ as following equations (1), (2) and (3).

$$
m \ddot{\eta}+c_{\eta} \dot{\eta}+k_{\eta} \eta=\frac{1}{2} \rho D U^{2}\left\{k H_{1}{ }^{*} \frac{\dot{\eta}}{U}+k H_{4}{ }^{*} \frac{\eta}{D / 2}\right\}
$$


where, $\rho$ is the air density $\left[\mathrm{kg} / \mathrm{m}^{3}\right], D$ is the diameter of cable model $[\mathrm{m}], U$ is the approaching wind velocity $[\mathrm{m} / \mathrm{s}], k$ is the reduced frequency $(=0.5 D \omega / U), \quad \omega$ is the circular frequency of forced vibration $(=2 \pi f), \eta$ is heaving response [m] and $H_{1}{ }^{*}, H_{4}{ }^{*}$ are aerodynamic derivatives.

$$
H_{1}^{*}=-\frac{1}{k} \frac{d C_{F}}{d \alpha}
$$

where, $\mathrm{d} C_{F} / \mathrm{d} \alpha$ is the slope of lateral force coefficient.

$S c=\frac{2 m \delta}{\rho D^{2}}=\frac{\pi}{2} H_{1}^{*}$

As shown in Fig.19, test results obtained from free-vibration tests and forced vibration tests show fairly good agreement with Saito criterion. As a matter of fact, wind tunnel data obtained by Saito using proto-type cables with 150mm diameter and approximately $10 \mathrm{~m}$ length in large-scale wind tunnel, are also associated to not divergent-type galloping but unsteady galloping. Saito describes to author's inquiry that $\operatorname{Vrcr}$ is also determined by the wind velocity when the peak-to-peak amplitude crosses $40 \%$ of cable diameter. Therefore, it can be concluded that Saito criterion at larger Scruton number in Sc-Vrcr should correspond to the unsteady galloping.

\section{0. $S c$-Vrcr characteristics of proto-type inclined stay-cables observed in the field}

Recently, some dry galloping phenomena, including violent cable vibration under precipitation, have been observed in Japan. The typical dry galloping occurred recently in Japan under without rain state as mentioned above. This cable-stayed bridge has curved bridge girder, therefore the longest stay cables are stayed directly on the ground. 
One of the longest cable showed violent vibration as shown in Fig.2, whose peak-to -peak amplitude is over than $1.5 \mathrm{~m}$ and it hit the girder. Then, the bridge girder, handrail and stay cable are seriously damaged.

Then, four observed cases which considered as the dry galloping are discussed including the previous example. One of the examples is a large scale elastic cable model with 30m length in the field, structural dynamics and climate conditions were comparatively verified. For these four cases, their cable vibrations seem to be divergent-type galloping because of their significantly large amplitude. These data are plotted on the Sc-Vrcr diagram by use of measured structural dynamic data, as shown in Fig.20. It seems that these data look to fit to Saito criteria. However, there are uncertainties on evaluation of their structural damping. For Bridge A, by inspection after violent cable vibration, it was found that the installed oil damper was totally damaged, as shown in Fig.21. But the same oil dampers installed to another stay cables which did not vibrate showed oil leakage. Therefore, it is natural to make estimation of that the installed oil damper had already been out of order as a damping device before the dry galloping occurred. If so, structural damping of cables should be much smaller than indicated in Fig.21. For the other two cases, which are Bridge B and Bridge C, measured structural damping of cables seems to be unexpectedly large. Because structural damping of another stay cable showed much smaller value such as $\delta=0.003$ or 0.005 from the vibration tests carried out on the same day. Therefore, if as the cable damping of these three cases $\delta=0.005$ would be used, which is mostly reasonable value for general stay-cables, their Scruton numbers are revised as plots on Sc-Vrcr in Fig.22. And it shows fairly good agreement with FHWA criterion. Therefore, FHWA criterion on $\mathrm{Sc}$-Vrcr diagram should correspond to divergent galloping. 


\section{Conclusions}

In conclusion, basing on various test results, it is implied that the dry galloping is caused by mitigation of Karman vortex shedding. This is fundamentally identical mechanism of galloping by interruption of communication between two separated flows, motion-induced self-excited vibration associated with low speed galloping of rectangular cylinder, or galloping of circular cylinder with splitter plate in near wake, which are pointed out by Nakamura.

Furthermore, galloping might be classified into steady galloping including divergent-type galloping with steady amplitude and unsteady galloping with unstable amplitude, which depend on Karman vortex mitigation level. In Sc-Vrcr diagram, FHWA criterion and Saito criterion correspond to steady galloping and unsteady galloping, respectively.

However, for clarification of general characteristics of Karman vortex on galloping instability should be waited more for further studies.

\section{References}

Bearman, P.W., Trueman D.M., 1972. An investigation of the flow around rectangular cylinders. The Aeronautical Quarterly, 23, 229-237.

Cheng, S., Irwin, P.A., Jakobsen, J.B., Lankin, J., Larose, G.L., Savage, M.G., Tanaka, H., Zurell, C., 2003. Divergent motion of cables exposed to skewed wind. In: Proceedings of the 5th Int. Symposium on Cable Dynamics, 271-278. 
FHWA/HNTB, 2005. Wind induced vibration of stay cables. Interim final report, RDT 05-004.

Larose, G.L., Jakobsen, J.B., Savage, M.G., 2003. Wind-tunnel experiments on an inclined and yawed stay cable model in the critical Reynolds number range. In: Proceedings of the 5th Int. Symposium on Cable Dynamics, 279-286.

Macdonald, J.H.G., 2005. Quasi-steady analysis of 2DOF inclined cable galloping in the critical Reynolds number range. In: Proceedings of the 6th International Symposium on Cable Dynamics, 435-442.

Matsumoto, M., 1998. Observed behavior of prototype cable vibration and its generation mechanism. In: Bridge Aerodynamics, Proceedings of the International Symposium on Advances in Bridge Aerodynamics, 189-211.

Matsumoto, M., 2000. Vortex-excited vibration and galloping instability of inclined cables. In: Proceedings of the 6th Italian Conference on Wind Engineering, 18-21.

Matsumoto, M., Hikami, Y., Kitazawa, M., 1994. Cable vibration and its aerodynamic mechanical control. In: Proceedings of the International Conference IABSE, Vol.2, 439-452.

Matsumoto, M., Knisely, C.W., Shiraishi, N., Kitazawa, M., Saitoh, T., 1989. Inclined-cable aerodynamics. In: Proceedings of Structural Design, Analysis \& Testing, Structures Congress '89, ASCE, 81-90.

Matsumoto, M., Shiraishi, N., Kitazawa, M., Knisely, C.W., Shirato, H., Kim, Y., Tsuji, M., 1990. Aerodynamic behavior of iclined circular clinders -cable aerodynamics-. In: Bluff Bodies Aerodynamics and its Applications, Elsevier, 63-72. 
Matsumoto, M., Shiraishi, N., Shirato, H., 1992. Rain-wind induced vibration of cables of cable-stayed bridges. Journal of Wind Engineering and Industrial Aerodynamics, 41-44, 2011-2022.

Matsumoto, M., Yagi, T., Hatsuda, H., Shima, T., Tanaka, M., 2007. Sensitivity of dry-state galloping of cable stayed bridges to Scruton number. In: Proceedings of the 7th International Symposium on Cable Dynamics, 331-338.

Matsumoto, M., Yagi, T., Liu, Q., Oishi, T., Adachi, Y., 2005. Effects of axial flow and Karman vortex interference on dry-state galloping of inclined stay-cables. In: Proceedings of the 6th International Symposium on Cable Dynamics, 247-254.

Matsumoto, M., Yokoyama, K., Miyata, T., Fujino, Y., Yamaguchi, H., 1989. Wind-induced cable vibration of cable-stayed bridges in Japan. In: Proceedings of Canada-Japan Joint Workshop on Bridge Aerodynamics, 101-110.

Nakamura, Y., Hirata, K., 1994. The Aerodynamic Mechanism of Galloping. Transaction of the Japan Society for Aeronautical and Space Science, Vol.36, No.114, 257-269.

Nakamura, Y., Hirata, K., Urabe, T., 1991. Galloping of rectangular cylinders in the presence of a splitter plate. Journal of Fluids and Structures, 5, 521-549.

Saito, T., Matsumoto, M., Kitazawa, M., 1994. Rain-wind excitation of cables on cable-stayed Higashi-Kobe Bridge and cable vibration control. In: Proceedings of the International Conference A.I.P.C.-F.I.P. Cable-Stayed and Suspension Bridges, 507-514.

Schewe, G., 1983. On the force fluctuations acting on a circular in crossflow from subcritical up to transcritical Reynolds numbers. Journal of Fluid Mechanics, 133, 265-285. 


\section{Figure captions}

Fig. 1: Comparison of wind velocity-damping relation of inclined dry cable (FHWA/HNTB, 2005).

Fig. 2: Violent cable vibration observed at certain proto-type cable-stayed bridge in Japan. (courtesy of Mr. H. Yoshikawa)

Fig. 3: Axial flow velocity (Va) in a wake of yawed cable model. ( $V=8 \mathrm{~m} / \mathrm{s}, \beta=45^{\circ}$, in smooth flow)

Fig. 4: Visualized axial flow by light strings for a proto-type cable.

Fig. 5: Axial flow velocity and approaching wind velocity of a stay-cable. $\left(\beta^{*}=40^{\circ}-50^{\circ}\right.$, where $\beta^{*}$ : equivalent yawing angle (Matsumoto, Knisely et al, 1989))

Fig. 6: Article axial flow generator.

Fig. 7: Galloping appearance for non-yawed/inclined cable with artificial axial flow.

Fig. 8: Velocity - amplitude diagrams in various cable-end conditions. ( $\beta=45^{\circ}, D=50 \mathrm{~mm}$, in smooth flow)

Fig. 9: Wind force coefficients for a cable model with surface roughness. ( $\beta=0^{\circ}$, $D=158 \mathrm{~mm}$, in smooth flow)

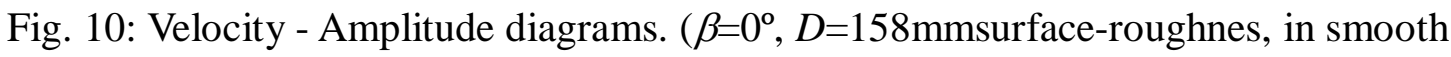
flow)

Fig. 11: Aerodynamic derivative $H_{1}{ }^{*}$ with surface roughness. $\left(\beta=0^{\circ}, 2 \eta=10 \mathrm{~mm}\right.$, $D=158 \mathrm{~mm}$, in smooth flow)

Fig. 12: Rivulet position effect on Velocity - Amplitude diagrams of non-yawed, yawed cable model. ( $D=54 \mathrm{~mm}$, in smooth flow)

Fig. 13: Water rivulet on prototype scale cable model during rain-wind induced vibration. ( $V=10 \mathrm{~m} / \mathrm{s})$ 
Fig. 14: Characteristics a perforated splitter plate. (P.R.: perforated ratio)

Fig. 15: Velocity - amplitude diagrams with various perforated splitter plate. $\left(\beta=0^{\circ}\right.$, $D=50 \mathrm{~mm}$, in smooth flow. P.R.: perforated ratio)

Fig. 16: Free vibration test results used cable model with smooth surface. $\left(\beta=45^{\circ}, D=54 \mathrm{~mm}\right.$, $S_{c}=1.22$, in smooth flow, 200mm window)

Fig. 17: Velocity - amplitude diagrams. $\left(\beta=45^{\circ}, D=50 \mathrm{~mm}, L=1400\right.$, in smooth flow, 200mm window)

Fig. 18: PSD of lift force obtained by forced vibration tests. $\left(\beta=0^{\circ}, D=100 \mathrm{~mm}, f=2.5 \mathrm{~Hz}\right.$, $U=2.0 \mathrm{~m} / \mathrm{s})$

Fig. 19: Comparison of galloping onset velocity. ( $\beta=45^{\circ}$, Smooth cable, in smooth flow)

Fig. 20: Field observation data at proto-type stay cable of cable-stayed bridges.

Fig. 21: Damaged oil damper.

Fig. 22: Field observation data at proto-type stay cable of cable-stayed bridges. 
Fig. 1: Comparison of wind velocity-damping relation of inclined dry cable (FHWA/HNTB, 2005).

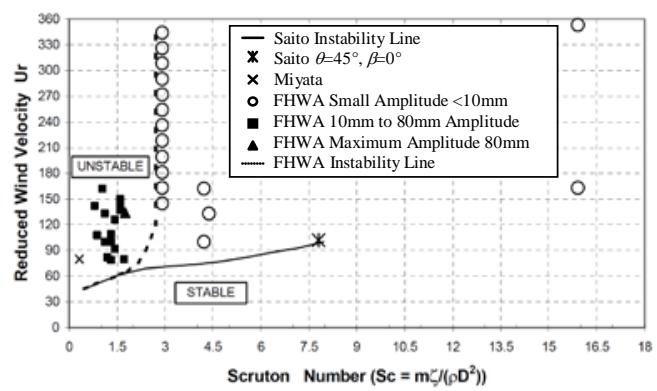


Fig. 2: Violent cable vibration observed at certain proto-type cable-stayed bridge in Japan.

(courtesy of Mr. H. Yoshikawa)
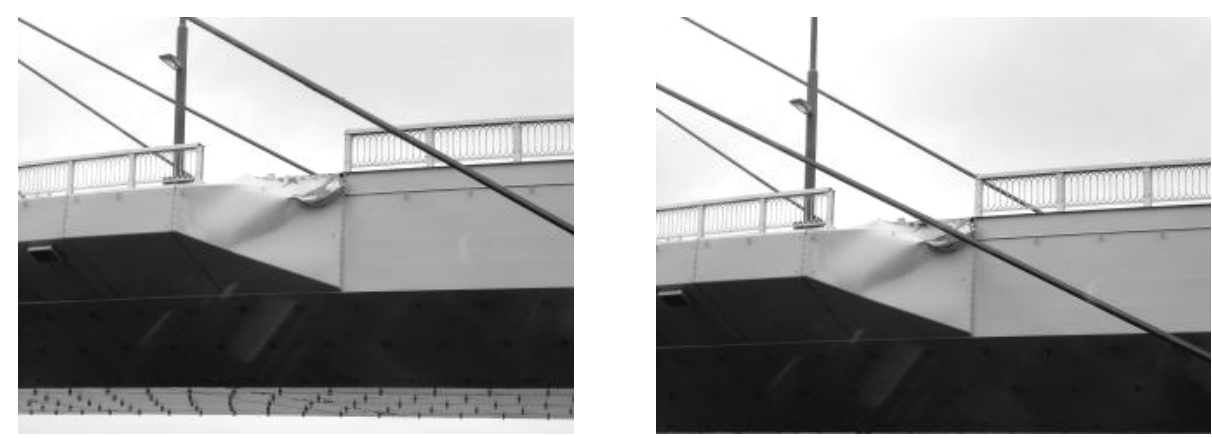
Fig. 3: Axial flow velocity ( $\mathrm{Va}$ ) in a wake of yawed cable model.

$\left(V=8 \mathrm{~m} / \mathrm{s}, \beta=45^{\circ}\right.$, in smooth flow)
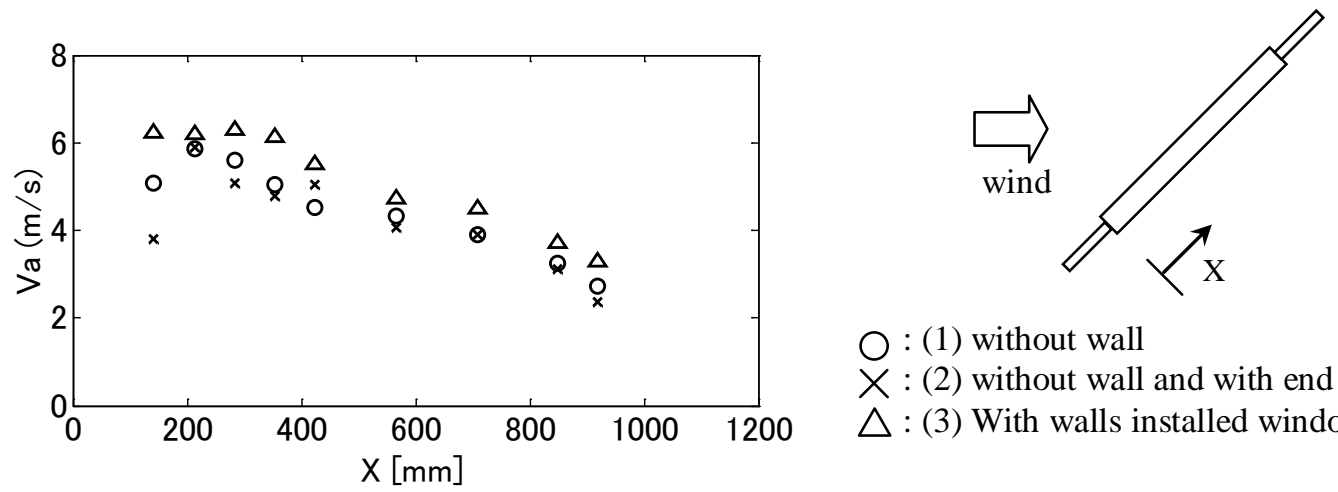

$\bigcirc:$ (1) without wall

$X:$ (2) without wall and with end plates

$\triangle$ : (3) With walls installed windows 
Fig. 4: Visualized axial flow by light strings for a proto-type cable.

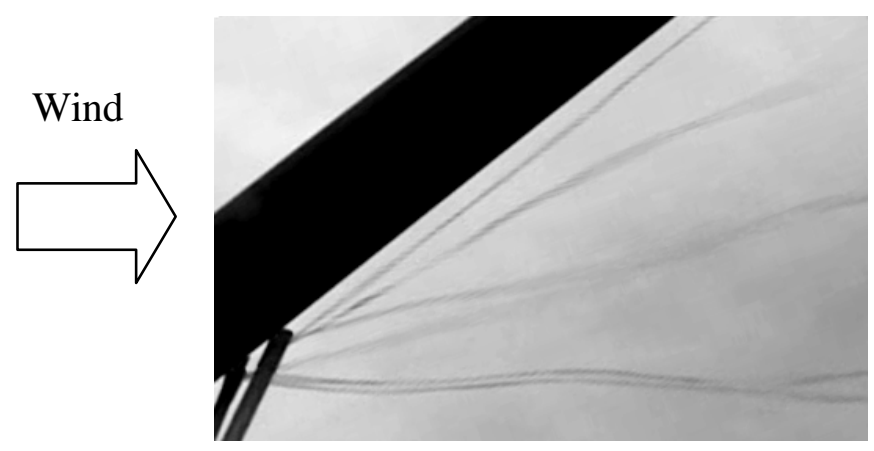


Fig. 5: Axial flow velocity and approaching wind velocity of a stay-cable.

$\left(\beta^{*}=40^{\circ}-50^{\circ}\right.$, where $\beta^{*}$ : equivalent yawing angle (Matsumoto, Knisely et al, 1989))

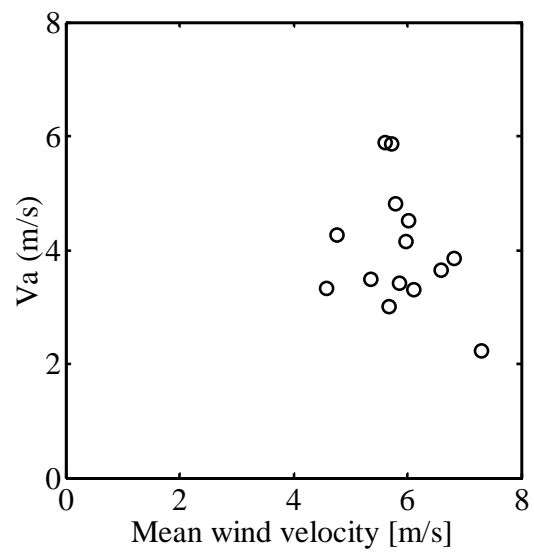


Fig. 6: Article axial flow generator.

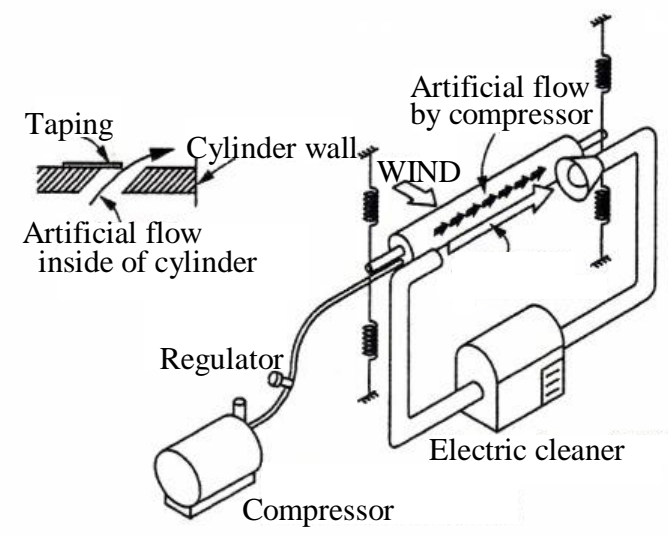


Fig. 7: Galloping appearance for non-yawed/inclined cable with artificial axial flow.

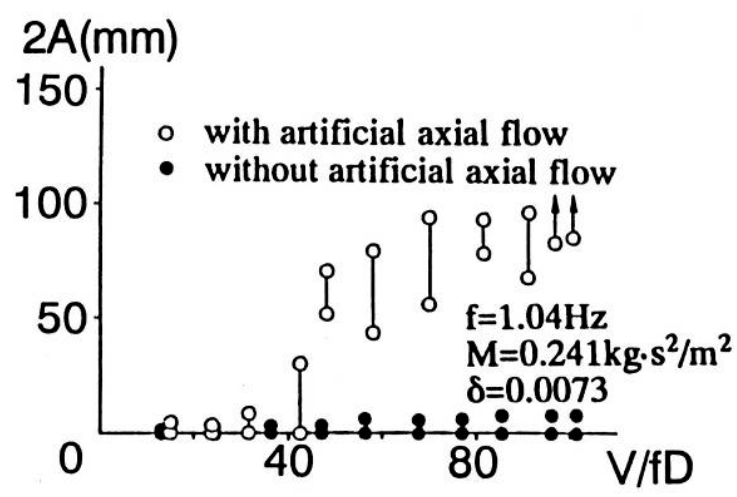


Fig. 8: Velocity - amplitude diagrams in various cable-end conditions.

( $\beta=45^{\circ}, D=50 \mathrm{~mm}$, in smooth flow)

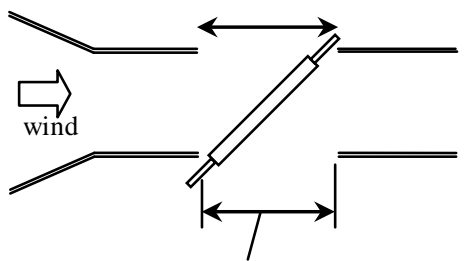

Walls without/with windows

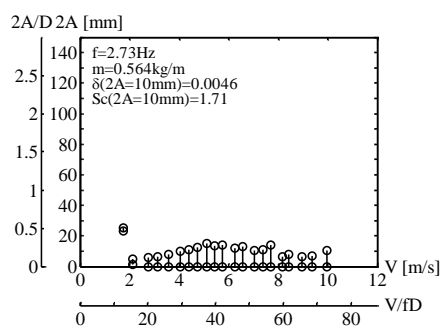

(2) without wall and with end plates

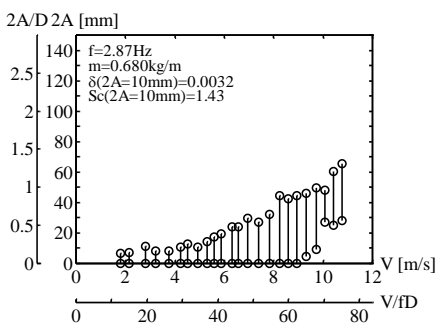

(1) without wall

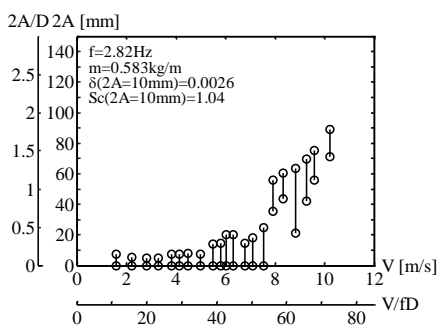

(3) with walls installed windows ( $\phi=170 \mathrm{~mm})$ 
Fig. 9: Wind force coefficients for a cable model with surface roughness.

( $\beta=0^{\circ}, D=158 \mathrm{~mm}$, in smooth flow)

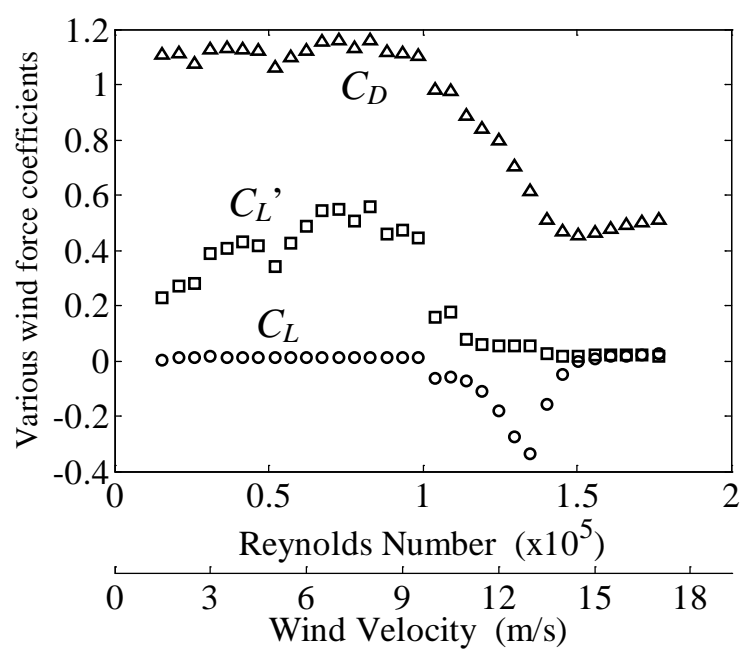




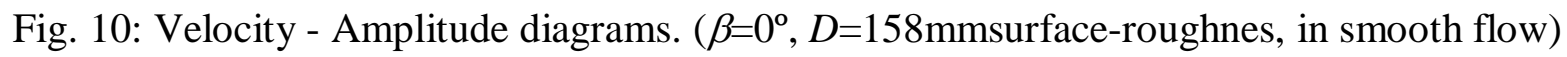

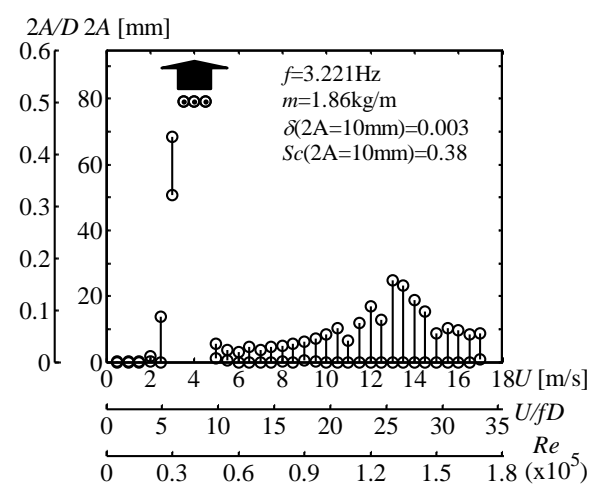


Fig. 11: Aerodynamic derivative $H_{1}{ }^{*}$ with surface roughness.

( $\beta=0^{\circ}, 2 \eta=10 \mathrm{~mm}, D=158 \mathrm{~mm}$, in smooth flow)
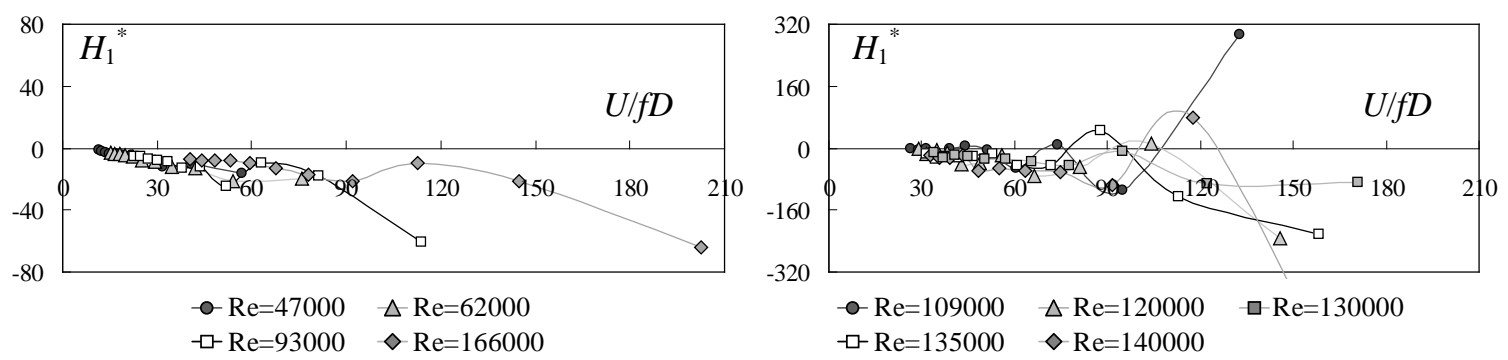
Fig. 12: Rivulet position effect on Velocity - Amplitude diagrams of non-yawed, yawed cable model. ( $D=54 \mathrm{~mm}$, in smooth flow)
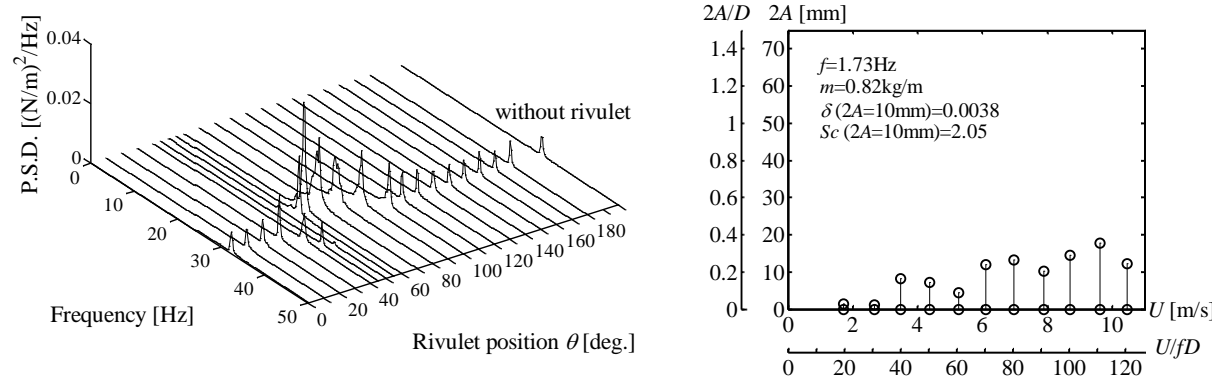

P.S.D. of the fluctuating lift force on stationary cable model $(U=6.0 \mathrm{~m} / \mathrm{s})$

$\theta=54^{\circ}$
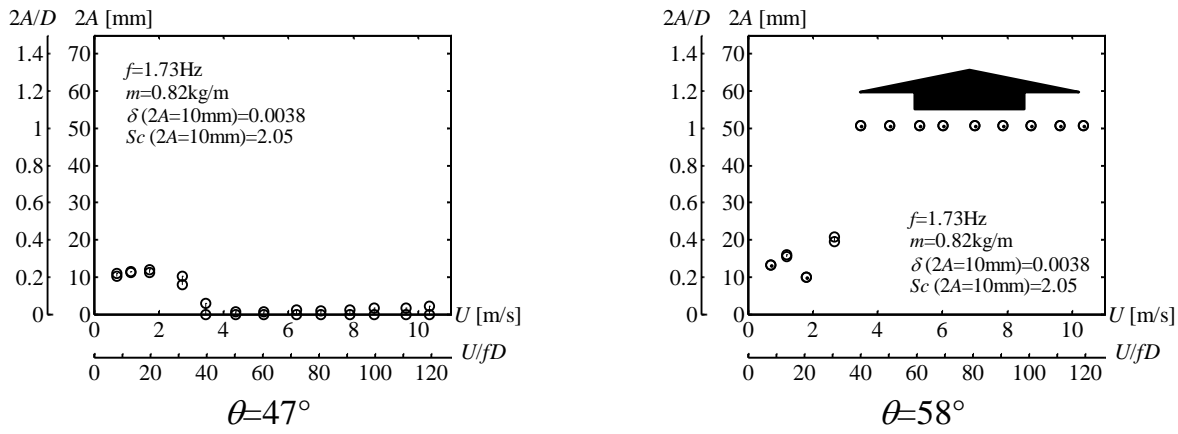

(a) $\beta=0^{\circ}$
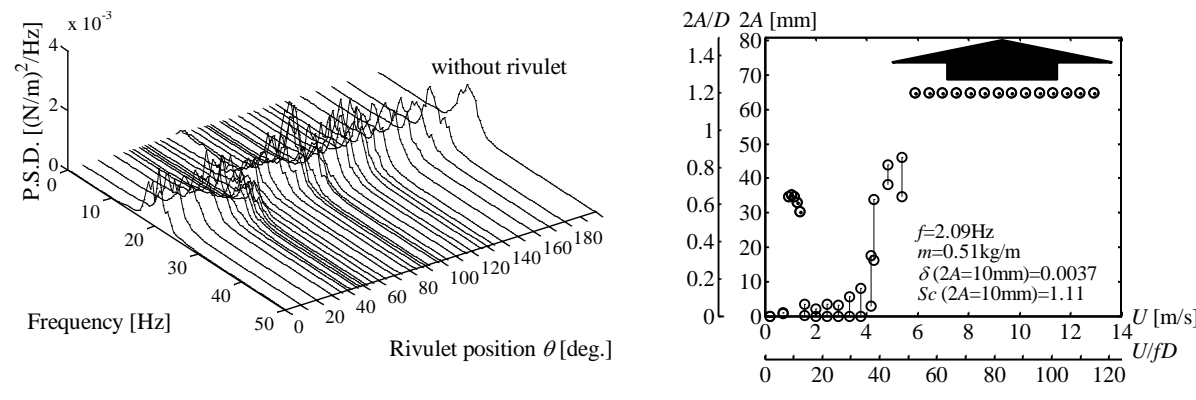

P.S.D. of the fluctuating lift force on stationary cable model $(U=6.0 \mathrm{~m} / \mathrm{s})$

$\theta=66^{\circ}$
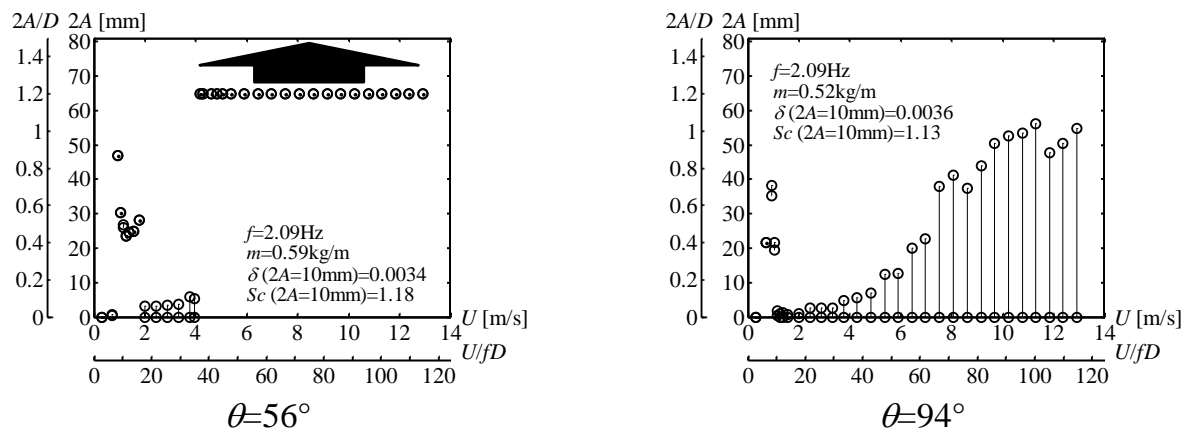

(b) $\beta=45^{\circ}$ 
Fig. 13: Water rivulet on prototype scale cable model during rain-wind induced vibration.

$(V=10 \mathrm{~m} / \mathrm{s})$

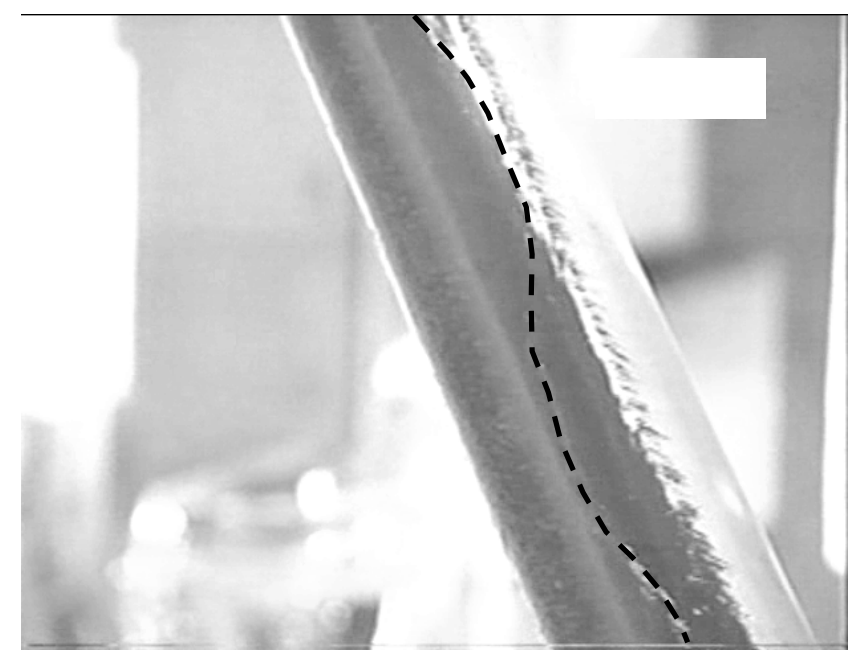


Fig. 14: Characteristics a perforated splitter plate. (P.R.: perforated ratio)

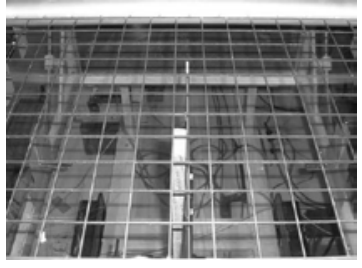

P.R. $90 \%$

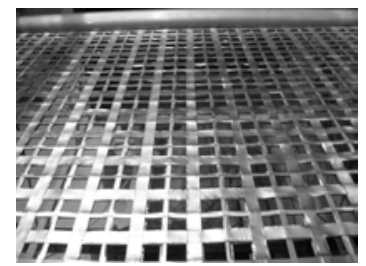

P.R. $40 \%$

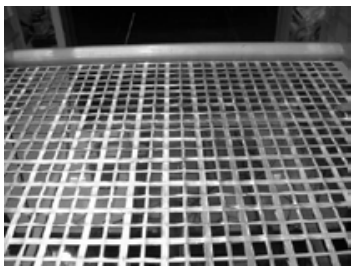

P.R. $60 \%$

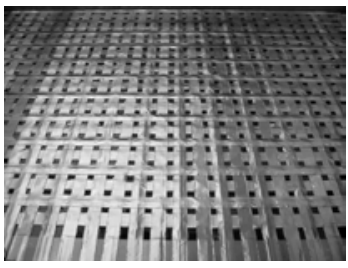

P.R. $10 \%$

(a) Perforated splitter plates (View from the downstream side)

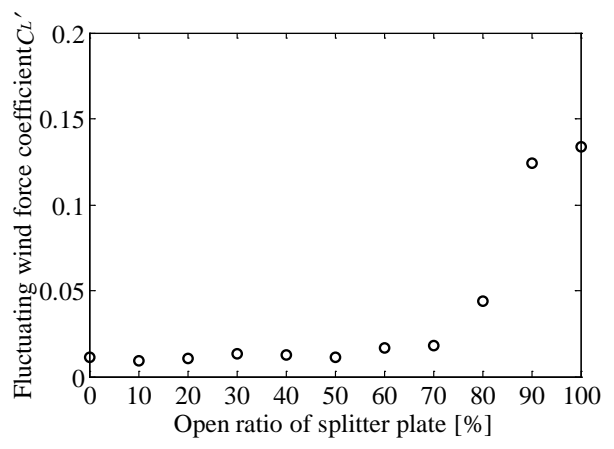

(b) Fluctuating lift force coefficient of the cable model $\left(\beta=0^{\circ}, U=6.0 \mathrm{~m} / \mathrm{s}\right)$ 
Fig. 15: Velocity - amplitude diagrams with various perforated splitter plate. ( $\beta=0^{\circ}, D=50 \mathrm{~mm}$,

in smooth flow. P.R.: perforated ratio)
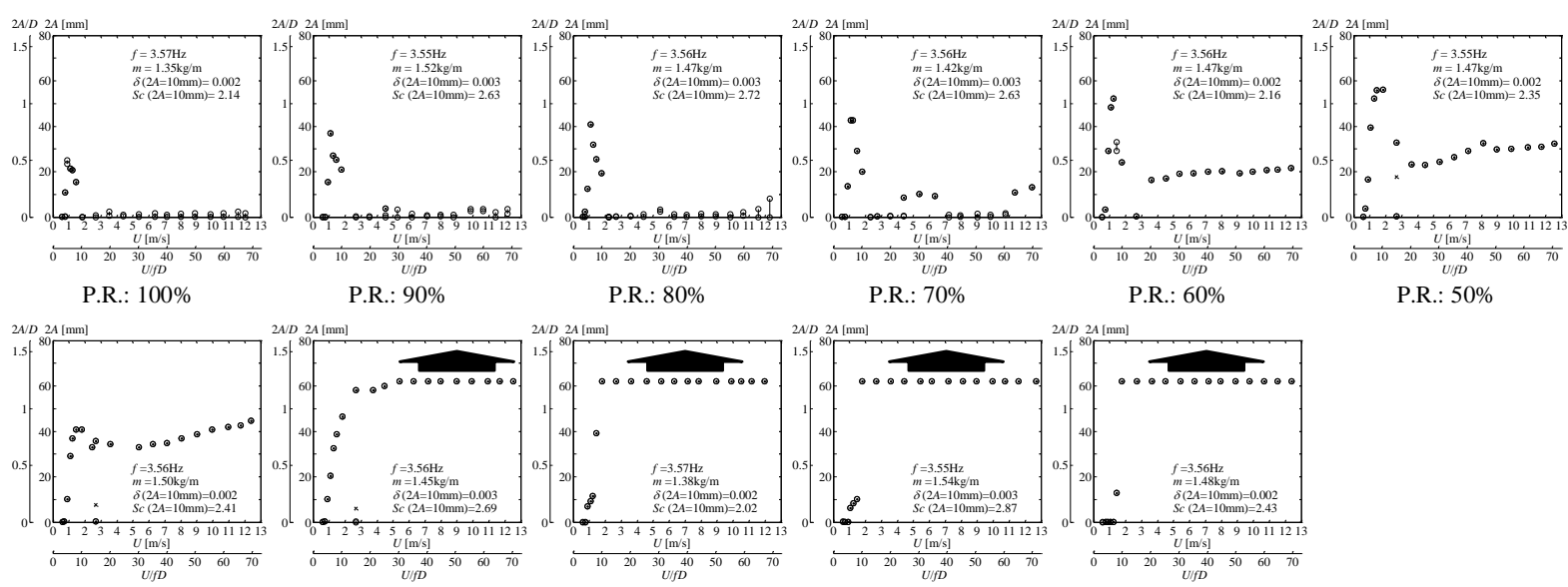

P.R.: $60 \%$

P.R.: $50 \%$

P.R.: $40 \%$

P.R.: $30 \%$

P.R.: $20 \%$

P.R.: $10 \%$

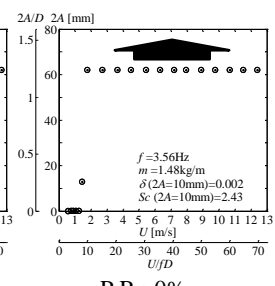

P.R.: $0 \%$ 
Fig. 16: Free vibration test results used cable model with smooth surface. $\left(\beta=45^{\circ}, D=54 \mathrm{~mm}\right.$,

$S c=1.22$, in smooth flow, $200 \mathrm{~mm}$ window)

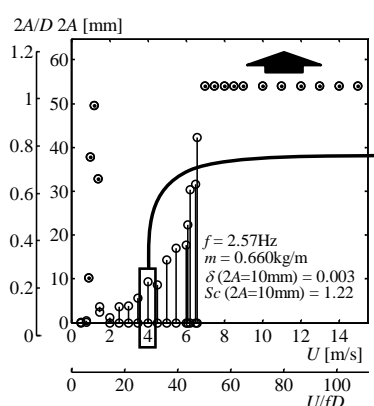

(a) V-A diagram

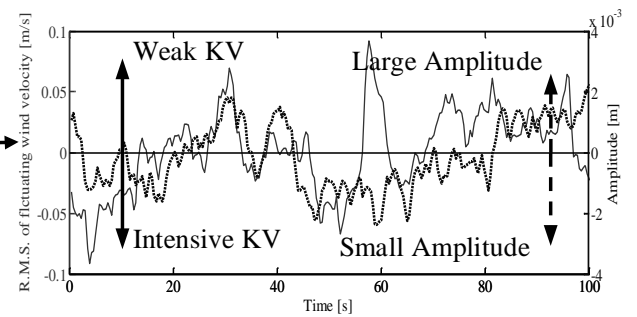

— : R.M.S. (5 [sec.]) of fluctuating wind velocity ( $f_{\mathrm{k}}$ B.P.F.)

.... : Amplitude of cross-flow response ( $f_{0}$ B.P.F.)

(b) Time history of fluctuating velocity and amplitude ( $U=4.0 \mathrm{~m} / \mathrm{s})$ 
Fig. 17: Velocity - amplitude diagrams. ( $\beta=45^{\circ}, D=50 \mathrm{~mm}, L=1400$, in smooth flow, $200 \mathrm{~mm}$ window)

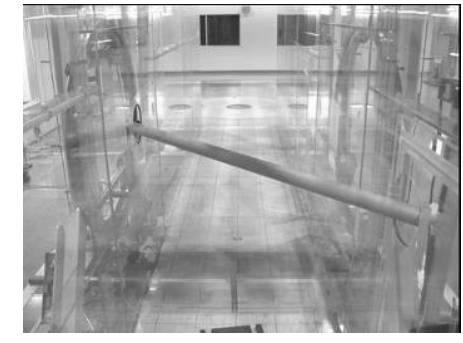

Set up for cable model

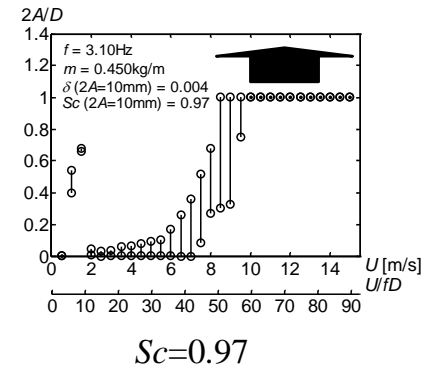

$S_{C}=0.97$
$S C=9.89$

$S_{C}=118$
Sc $=25.9$

$S_{c}=158$ 
Fig. 18: PSD of lift force obtained by forced vibration tests. $\left(\beta=0^{\circ}, D=100 \mathrm{~mm}, f=2.5 \mathrm{~Hz}\right.$, $U=2.0 \mathrm{~m} / \mathrm{s})$

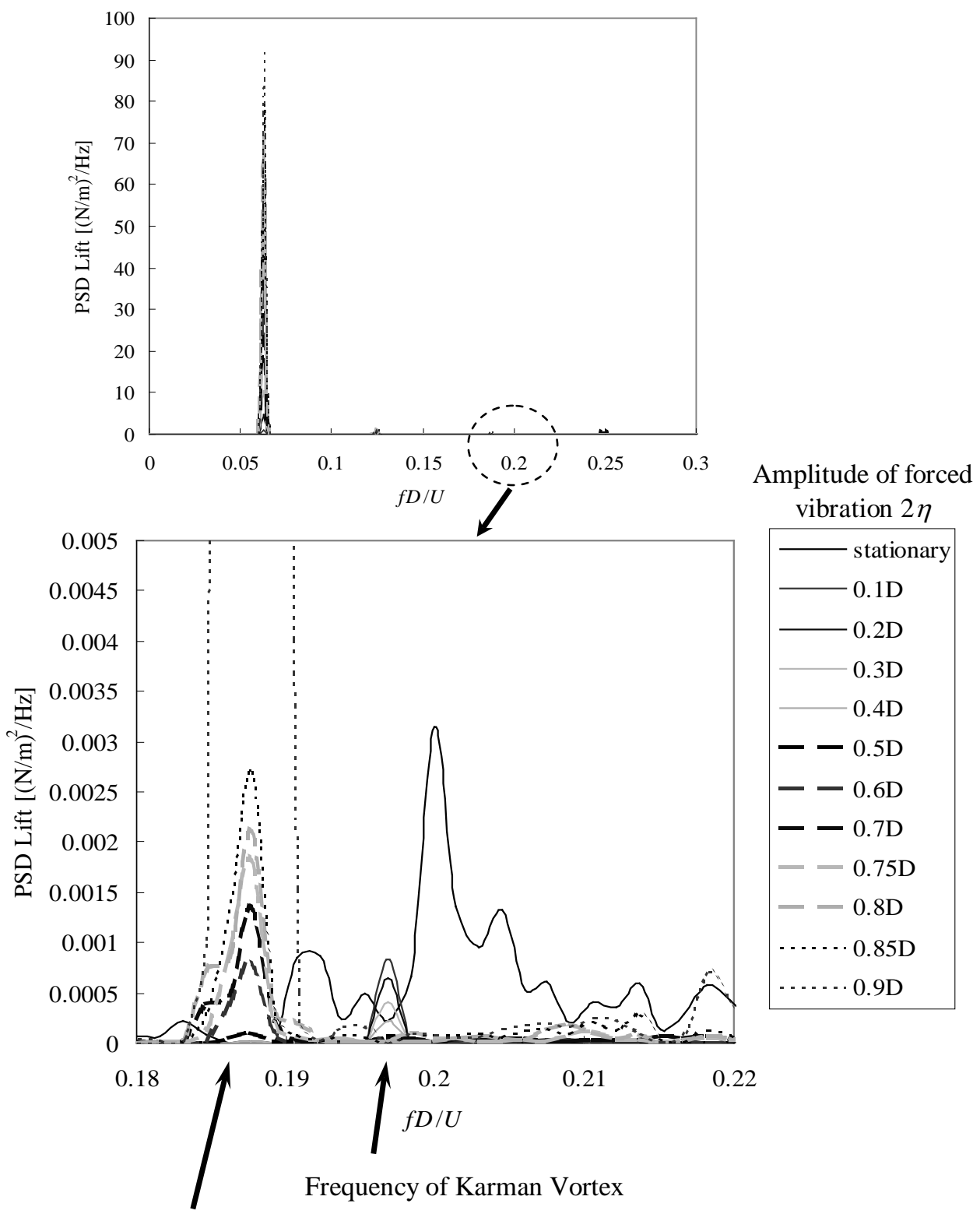

Frequency of forced vibration 
Fig. 19: Comparison of galloping onset velocity. ( $\beta=45^{\circ}$, Smooth cable, in smooth flow)

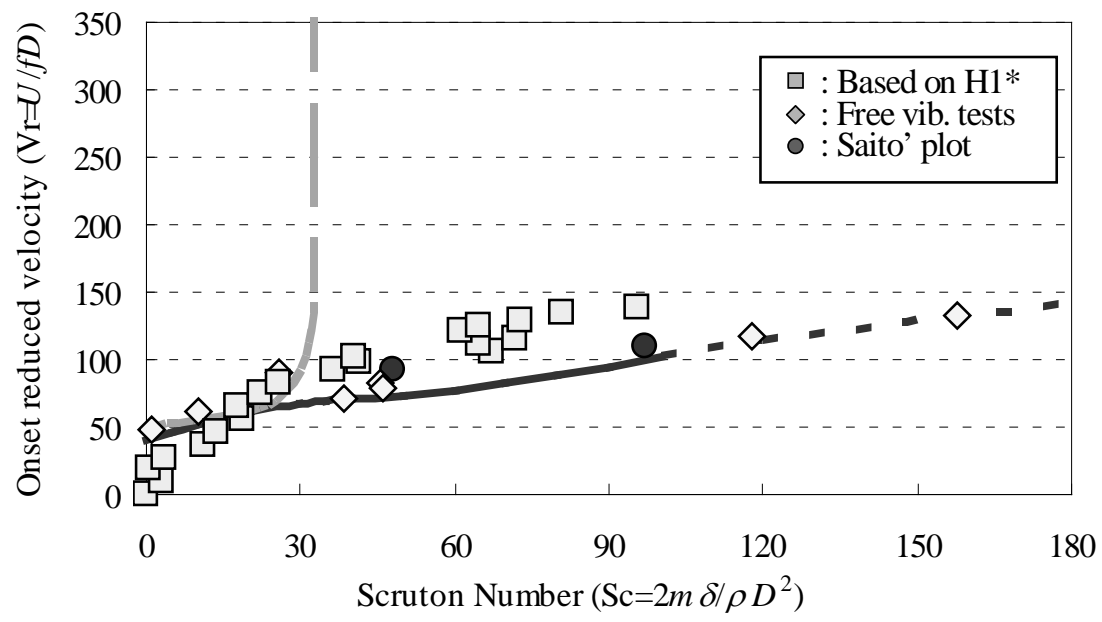


Fig. 20: Field observation data at proto-type stay cable of cable-stayed bridges.

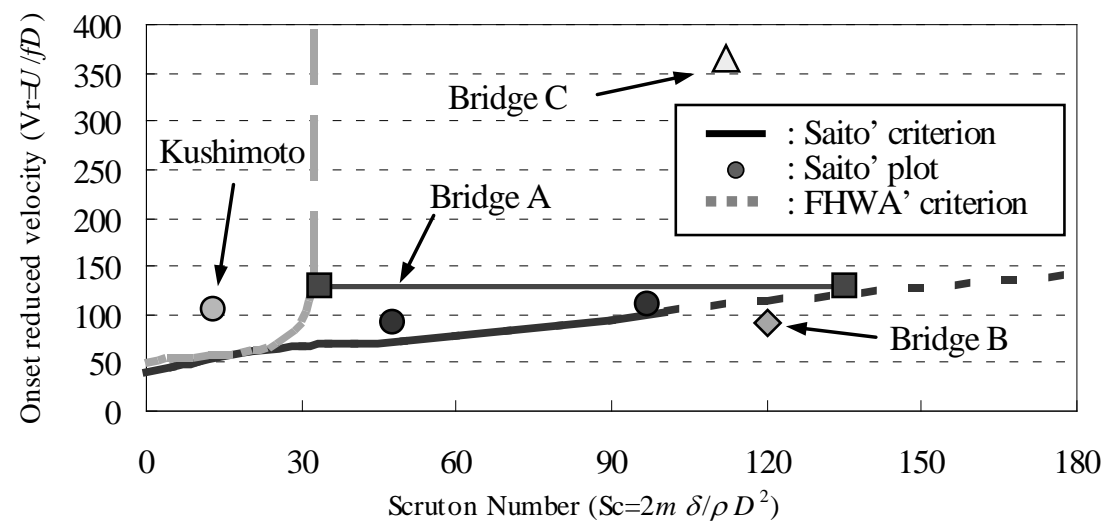


Fig. 21: Damaged oil damper.

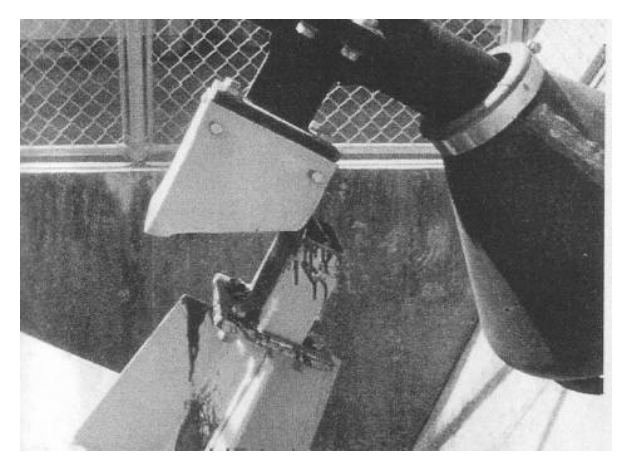


Fig. 22: Field observation data at proto-type stay cable of cable-stayed bridges.

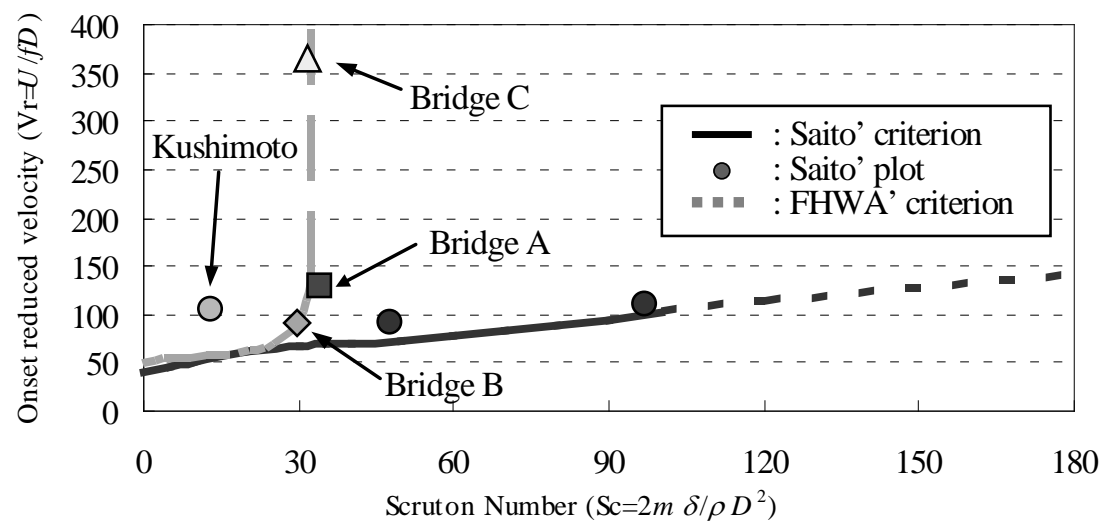

